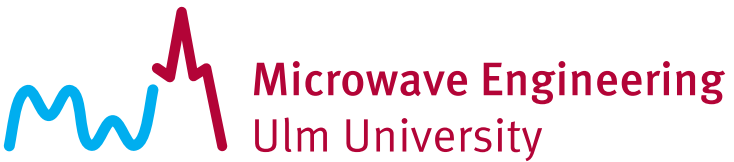

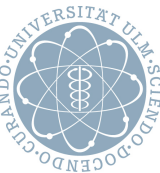

\section{Position Acquisition for a Multicopter-Based Synthetic Aperture Radar}

\author{
Markus Schartel, Rik Bähnemann, Ralf Burr, Winfried Mayer, and Christian Waldschmidt
}

(C) 2019 IEEE. Personal use of this material is permitted. Permission from IEEE must be obtained for all other uses, in any current or future media, including reprinting/republishing this material for advertising or promotional purposes, creating new collective works, for resale or redistribution to servers or lists, or reuse of any copyrighted component of this work in other works. 


\title{
Position Acquisition for a Multicopter-Based Synthetic Aperture Radar
}

\author{
Markus Schartel*1, Rik Bähnemann ${ }^{2}$, Ralf Burr ${ }^{3}$, Winfried Mayer ${ }^{4}$, \\ and Christian Waldschmidt ${ }^{1}$
}

${ }^{1}$ Institute of Microwave Engineering, Ulm University, 89081 Ulm, Germany

*email: markus.schartel@uni-ulm.de

${ }^{2}$ Autonomous Systems Lab, ETH Zürich, 8092 Zürich, Switzerland

${ }^{3}$ Laboratory of Microtechnology, Ulm University of Applied Sciences, 89075 Ulm, Germany

${ }^{4}$ Endress+Hauser SE+Co. KG, 79689 Maulburg, Germany

\begin{abstract}
Humanitarian demining is still mainly carried out by hand. The most trusted and widely used technical tool is probably the metal detector. However, these sensors are hand-held devices which are operated closely to the surface. To make the process of mine clearance safer, a ground penetrating synthetic aperture radar (GPSAR) was developed that can be operated on an autonomous flying unmanned aerial system (UAS). A key challenge of this system approach is the accurate position acquisition of the UAS. This paper compares a real time kinematic global navigation satellite system (RTK GNSS) and a total station with respect to UAS-based synthetic aperture radar (SAR) image processing. First, the systems and the associated signal processing chain will be briefly presented, then the trajectories and the processed SAR image will be compared.
\end{abstract}

\section{Introduction}

Anti-personnel (AP) mines are a hidden and permanent threat to civilians in many countries throughout the world. In 2016, there were more than 8600 casualties recorded [1]. Blast mines are typically buried at shallow depths, and many of them are plastic-cased to make the detection difficult. Because of the low metallic content and the weak dielectric contrast to the surrounding soil, the reliable detection of mines is still a great technical challenge. In humanitarian demining the most trusted and widely used technical tool is probably the metal detector [2]. Due to the minimal metal content in modern blast mines, GPRs are a promising complement to metal detectors [3]. However, most commercial sensors are hand-held devices which are operated very closely to the surface of the ground.

Due to the recent progress in the field of multicopters, it is now possible to integrate the abovementioned sensors into a so-called unmanned aerial system (UAS). The use of an autonomous flying sensor platform enables the collection and analysis of data safely, before a deminer has to enter the hazardous environment. UAS-based approaches using a down-looking GPR for mine detection are presented in $[4,5]$. The area under investigation has to be scanned in parallel straight lines (B-scans) so that a high-resolution three-dimensional image (C-scan) can be

The 20th International Radar Symposium IRS 2019, June 26-28, 2019, Ulm, Germany 
generated. In contrast, when using a multi-static side-looking radar, the area throughput can be increased as demonstrated in [6]. The vehicle-based multiple-input multiple-output (MIMO) ground penetrating synthetic aperture radar (GPSAR) presented in this paper, however, is not suitable for a multicopter application due to its weight and size.

As a trade-off, a side-looking single-input single-output (SISO) GPSAR was developed that can be operated on board a multicopter. The key challenge of this approach is the high-precision localization required for interferometric SAR. In this paper the SAR imaging results using a real time kinematic global navigation satellite system (RTK GNSS) and a total station for position acquisition are compared. In Section II the position acquisition systems are introduced. The measurement system is briefly described in Section III. In Section IV the processing chain is explained. The measurement results are discussed in Section V, and Section VI gives a short conclusion.

\section{Position Acquisition}

The RTK GNSS shown in Fig. 1a consists of a base station and a rover station, which are connected via a radio link. The rover station is connected to the flight controller of the multicopter, which provides the position data via a serial interface. According to the specification, the RTK GNSS has a horizontal accuracy and a vertical accuracy of $\pm 10 \mathrm{~mm}$ and $\pm 20 \mathrm{~mm}$, respectively [7]. The position is updated at a rate of $5 \mathrm{~Hz}$ and the radio link can be used up to a distance of $7 \mathrm{~km}$.

The total station depicted in Fig. $1 \mathrm{~b}$ measures the distance, the elevation angle, and the azimuth angle to a reference point using a laser [8]. The total station can track a prism that is attached to the multicopter. In tracking mode, the accuracy of the distance measurement is $\pm 4 \mathrm{~mm}$ and the angular accuracy is $2^{\prime \prime}$, i.e. $0.56 \mathrm{~m}^{\circ}$. In other words, up to a distance of about $1031 \mathrm{~m}$, the horizontal and vertical accuracy of the total station is better than $\pm 10 \mathrm{~mm}$. The measurement rate is $20 \mathrm{~Hz}$ and the maximum range is $2500 \mathrm{~m}$.

Both measuring systems have their advantages and disadvantages. Assuming that the UAS flies at a maximum distance of $500 \mathrm{~m}$ during missions, the total station convinces with its high positioning accuracy. But obstacles in the line of sight cause the position acquisition to be interrupted. The usability in a field with many obstacles is therefore severely restricted. In addition, a highly accurate time synchronization between the computer that stores the data of the total station and the computer on board the multicopter is required.

On the other hand, the RTK GNSS solution has the advantage, that all the data can be recorded on the same computer on board the multicopter. Therefore, no additional time synchronization is required. Furthermore, obstacles in the line of sight between the base station and the rover do not lead to a failure of the position recording. Moreover, the price of the RTK GNSS employed is significantly lower than that of the total station. But a measuring rate of $5 \mathrm{~Hz}$ appears very slow for recording fast flight manoeuvres in 3D space. 


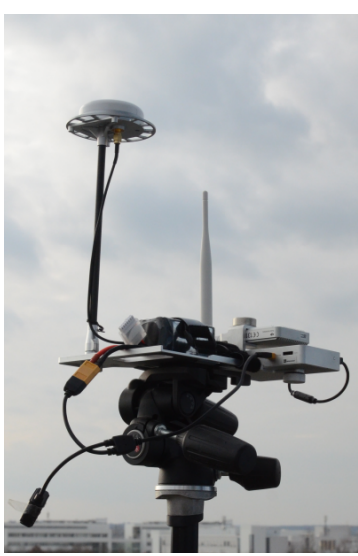

(a) RTK base station.

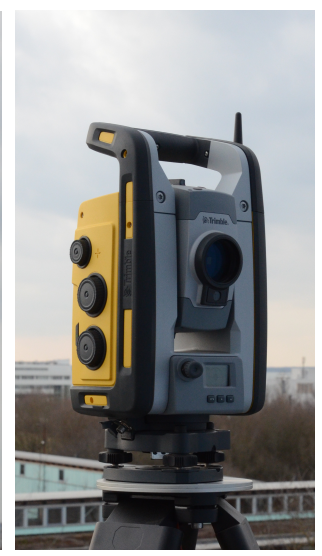

(b) Total station.

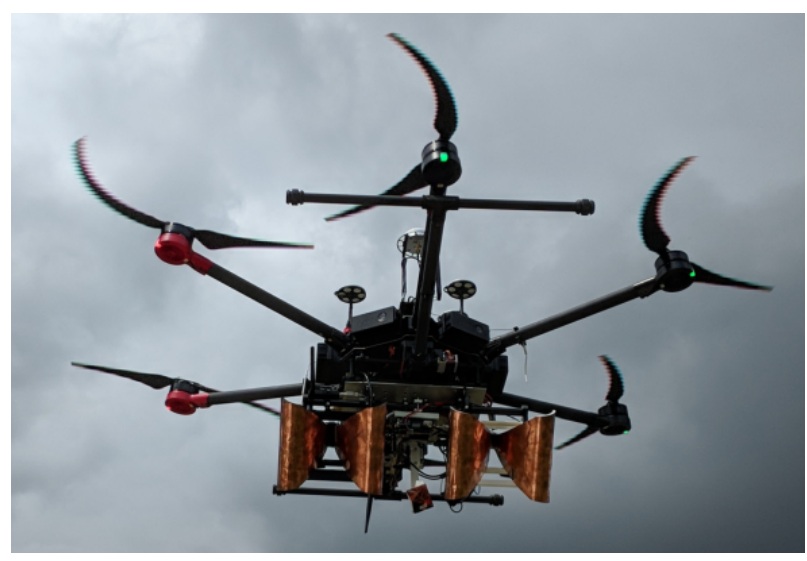

(c) UAS.

Figure 1: Measuring system consisting of an RTK base station, a total station, and the UAS. The UAS is equipped with an RTK GNSS rover station, a $360^{\circ}$-prism, a single-board computer for data storage, and the bistatic FMCW SAR.

\section{Measurement System}

The measurement system is shown in Fig. 1. It consists of a multicopter [9], an RTK GNSS [7], a total station [8] in combination with a $360^{\circ}$-prism, and a bi-static frequency-modulated continuous-wave (FMCW) SAR operating in the frequency range from $1 \mathrm{GHz}$ to $4 \mathrm{GHz}$. The radar has a chirp repetition frequency (CRF) of $30 \mathrm{~Hz}$ and is presented in [10]. The first UASbased SAR and GPSAR measurement results using the RTK GNSS to acquire the position are presented in $[11,12]$. The rotatable, lightweight 3D-printed horn antennas are introduced in [13]. The maximum flight time of the UAS is approximately $23 \mathrm{~min}$.

The output data of the RTK GNSS, the UAS-integrated inertial measurement unit (IMU), and the SAR are time-stamped and stored on a single-board computer on board the UAS. The time stamps of the data of the flight controller are based solely on the arrival time of the data packets at the single-board computer. There is no additional hardware handshake, e.g., by evaluating a pulse per second (PSS) signal for time synchronization. Due to the time required to process and transmit the data and unknown delays due to input and output buffers, minor timing errors can be expected.

The position data of the total station are time-stamped and stored on a laptop. The single-board computer and the laptop are time synchronised over a wireless local area network (WLAN) using a network time protocol (NTP) solution. Due to the operating principle of the NTP time synchronization, the drift-rate of the synchronized clock is sign-inverted at each synchronisation event to average out the temporal offset. The clocks drift apart until the next synchronization event. In consequence, the slave clock time oscillates around the master clock, leading to a variable although constrained residual error. 


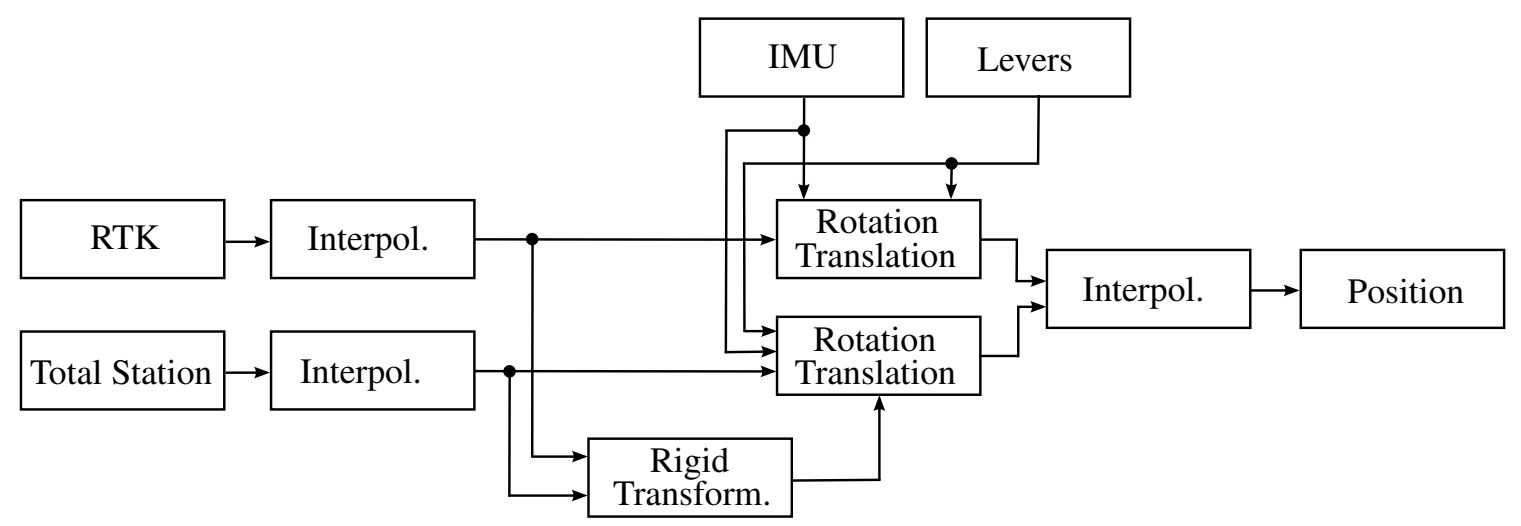

Figure 2: Calculation of the phase center position using the data of the RTK and the total station.

\section{Processing Chain}

For image formation a back-projection algorithm is used [14]. For each chirp, the phase center position of the radar antenna is required. As indicated in [11], the position must be known with an accuracy of $\pm 1 \mathrm{~cm}$ in $3 \mathrm{D}$ space. Larger deviations lead to a blurring of the target response.

In the following, the signal processing chain illustrated in Fig. 2 is explained in detail. The phase center is calculated from the position of the RTK GNSS or the total station using the IMU data and the appropriate lever arms. First, the RTK GNSS and the total station data are interpolated to match the timestamps of the IMU. Since the RTK GNSS and the total station acquire the data in a different coordinate system, the next step is to determine the angle of rotation and the translation between the two coordinate systems using the $x$ - and $y$-coordinates. Then, the IMU data and the lever arms between the sensors and the IMU are used to calculate the 3Dposition of the IMU. Afterwards, the IMU data and the lever arms between the IMU and the radar antenna are used to calculate the position of the phase center. The last step is to assign the interpolated phase center positions to the timestamps of the radar data.

No further filtering or signal processing of the position data was performed for the measurements presented in this paper.

\section{Measurement Results}

The performance of the position acquisition systems is particularly relevant for dynamic processes in 3D space. The systems are compared on the basis of the quality of SAR images using a simple measurement scenario shown in Fig. 3 and realistic trajectories as shown in Fig. 4.

In order to avoid interferences from buildings or trees (multipath effects), the measurements were carried out on a large open area. The minimum and maximum distance between the total station and the prism was $4 \mathrm{~m}$ and $35 \mathrm{~m}$, respectively. As reference 20 reflectors have been placed on the surface as shown in Fig. 3. The UAS was manually steered to perform 12 stripmap 


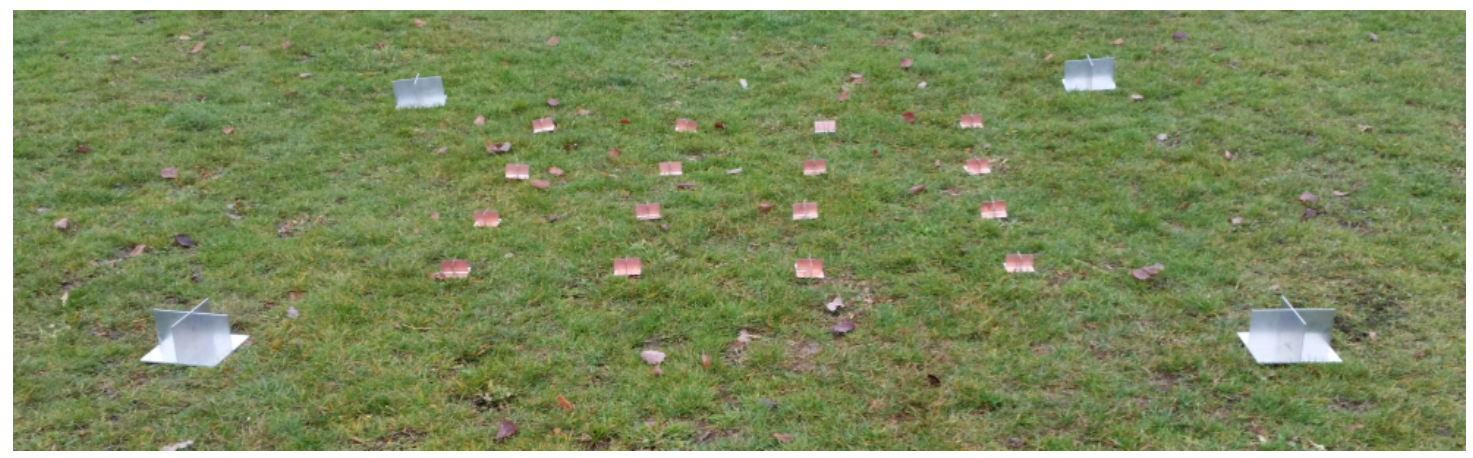

Figure 3: Photo of the measurement setup.

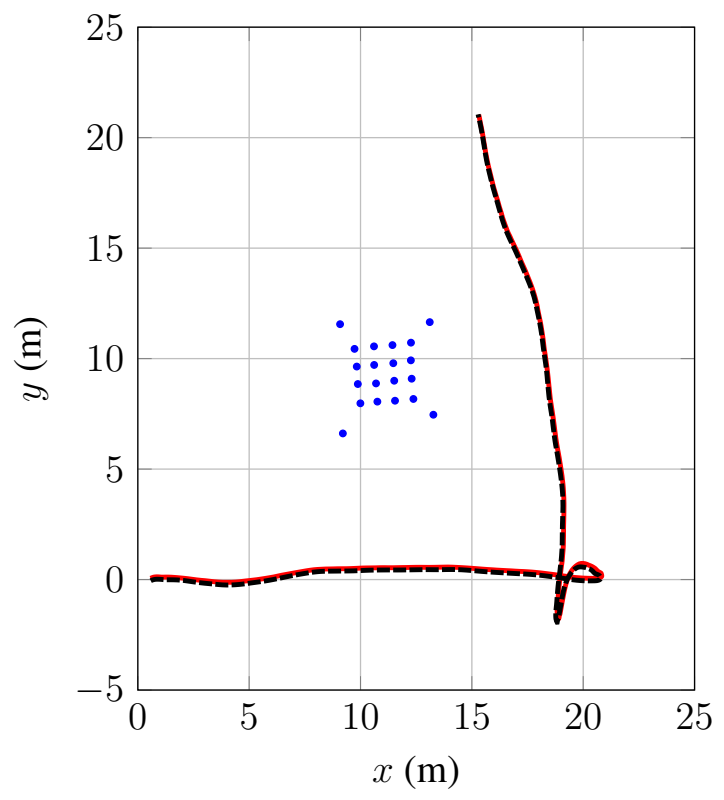

(a) $x y$-plane.
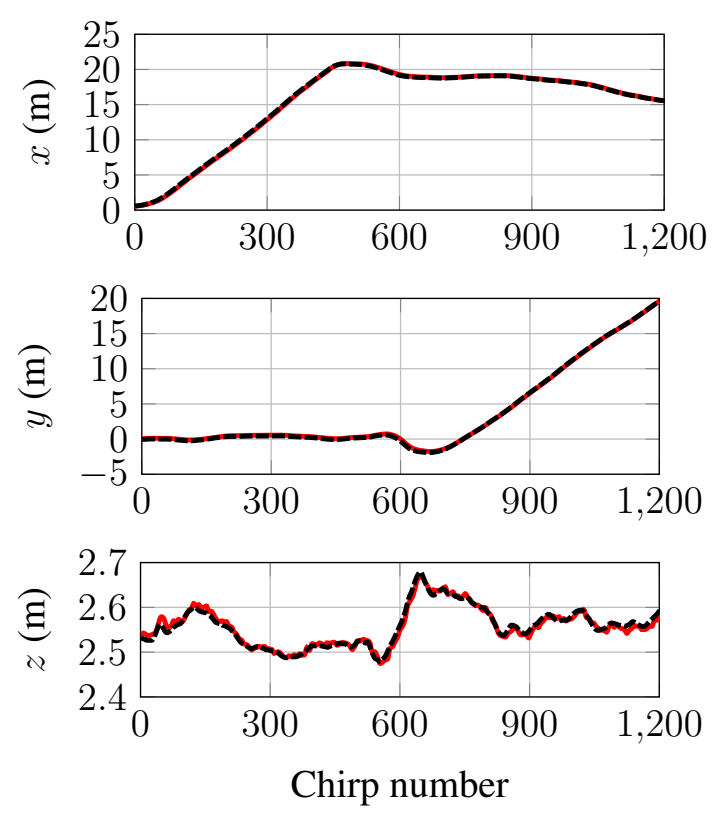

(b) Coordinates as a function of the chirp number.

Figure 4: Trajectory of the multicopter (L-shaped flight) measured with the RTK GNSS ( - ) and the total station (---). The positions of the reflectors are marked with (•).

and L-shaped SAR measurements at a constant airspeed of approximately $1 \mathrm{~m} / \mathrm{s}$.

After compensation the distance-dependent signal attenuation, the 12 single-look SAR images are combined by an incoherent addition as shown in Fig. 5. The measurement result shows, that the total station solution outperforms the RTK GNSS solution in terms of signal-to-noise ratio by $10 \mathrm{~dB}$. The degraded image quality can be explained by the lower position accuracy of the RTK GNSS and mainly by time synchronisation errors. It is not known when the GNSS data were valid. Due to the low update rate of $5 \mathrm{~Hz}$, an error of up to $20 \mathrm{~cm}$ can occur even at a slow airspeed of $1 \mathrm{~m} / \mathrm{s}$. As a result, the targets in each individual SAR image are displaced in the $x y$ plane, which in turn results in the target responses being strongly smeared after the incoherent addition. 


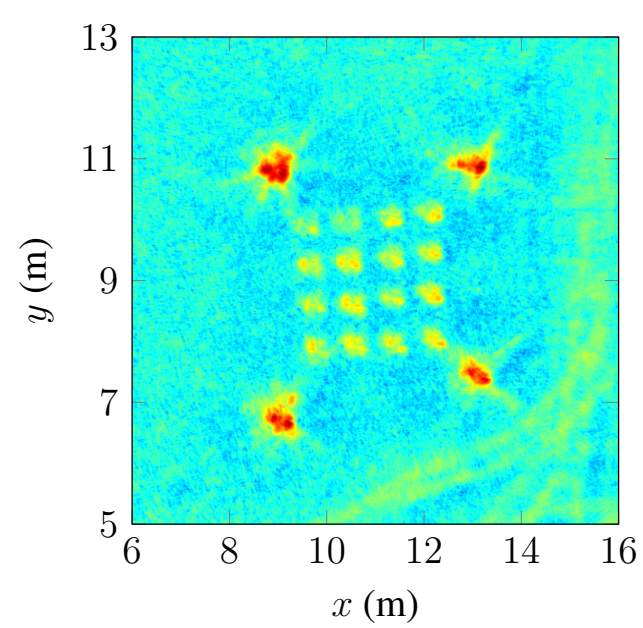

(a) RTK GNSS.

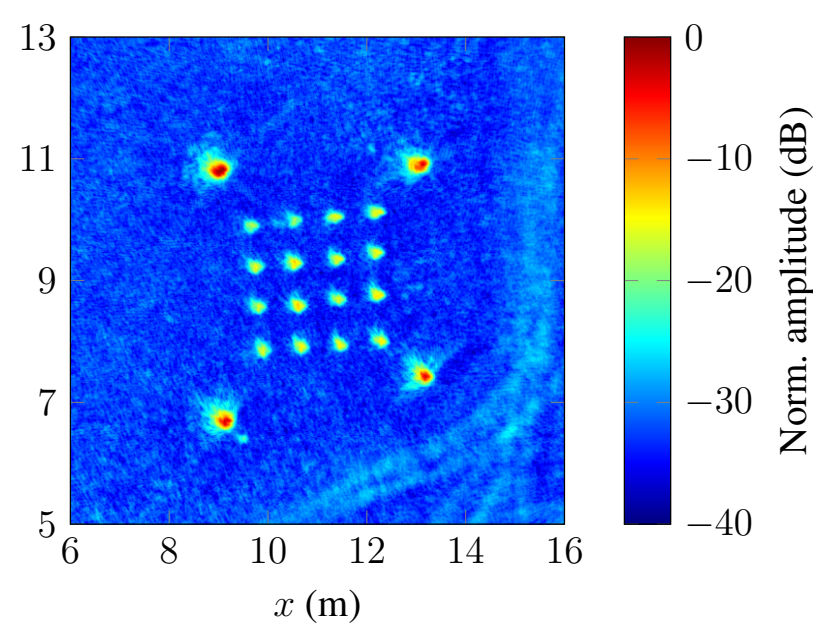

(b) Total station.

Figure 5: Incoherent superposition of 12 SAR images.

\section{Conclusion}

In this paper an RTK GNSS and a total station were compared to capture the position of a multicopter in 3D space. The superposition of several SAR images was used as the basis for the evaluation. The total station approach clearly outperforms the RTK GNSS solution in terms of image quality. Due to the price and the usability in the field, the measurement results with the total station can be considered as a reference to be achieved.

\section{Acknowledgement}

The authors would like to thank the Urs Endress Foundation for the support.

\section{References}

[1] International Campaign to Ban Landmines, Landmine Monitor 2017. Human Rights Watch.

[2] D. J. Daniels, "Mine Detection," in Ground Penetrating Radar 2nd Edition, Institution of Electrical Engineers, 2004.

[3] D. J. Daniels and P. Curtis, "MINETECT," in Proceedings of the 2nd International Workshop on Advanced Ground Penetrating Radar, May 2003, pp. 110-114.

[4] J. Colorado et al., "An integrated aerial system for landmine detection: SDR-based Ground Penetrating Radar onboard an autonomous drone," Advanced Robotics, vol. 31, no. 15, pp. 791-808, 2017.

[5] G. Fasano et al., "Proof of concept of micro-UAV-based radar imaging," in International Conference on Unmanned Aircraft Systems (ICUAS), Jun. 2017, pp. 1316-1323. 
[6] M. Peichl et al., "TIRAMI-SAR - a synthetic aperture radar approach for efficient detection of landmines and UXO," in European Conference on Synthetic Aperture Radar, Jun. 2014.

[7] DJI, "D-RTK Release Notes," 2017.

[8] Trimble, "Datasheet Trimble S9/S9 HP Total Station," 2018.

[9] DJI, "Matrice 600 Pro User Manual v1.0," 2017.

[10] R. Burr et al., "Design and Implementation of a FMCW GPR for UAV-based Mine Detection," in IEEE MTT-S International Conference on Microwaves for Intelligent Mobility (ICMIM), 2018.

[11] M. Schartel et al., "UAV-Based Ground Penetrating Synthetic Aperture Radar," in IEEE MTT-S International Conference on Microwaves for Intelligent Mobility, 2018.

[12] M. Schartel et al., "A Multicopter-Based Focusing Method for Ground Penetrating Synthetic Aperture Radars," in IEEE International Geoscience and Remote Sensing Symposium, Jul. 2018.

[13] R. Burr et al., "Lightweight Broadband Antennas for UAV based GPR Sensors," in 48th European Microwave Conference (EuMC), Oct. 2018.

[14] E. C. Zaugg and D. G. Long, "Generalized Frequency Scaling and Backprojection for LFM-CW SAR Processing," IEEE Transactions on Geoscience and Remote Sensing, vol. 53, no. 7, pp. 36003614, Jul. 2015. 